\title{
Computational Assessment of the Three-Dimensional Configuration of Dissolved Organic Matter Chromophores and Influence on Absorption Spectra
}

\author{
Elena A. Vialykh, ${ }^{\mathrm{a}}$ Garrett McKay, ${ }^{\mathrm{b}}$ and Fernando L. Rosario-Ortiz ${ }^{\mathrm{a}, \mathrm{c}^{*}}$ \\ aDepartment of Civil, Environmental, and Architectural Engineering \\ University of Colorado Boulder, CO 80309 \\ bZachry Department of Civil \& Environmental Engineering \\ Texas A\&M University, TX 77843 \\ ${ }^{c}$ Environmental Engineering Program \\ University of Colorado Boulder, CO 80309
}

*Corresponding author: Fernando.rosario@colorado.edu 


\section{Table of Contents}

Text S1. Chemical characteristics of SRFA used to build a model

Figure S1. Example of LogD solubility calculation for a molecule

Table S1. Molecular composition of the different models created and calculated values for $\operatorname{LogD}$ and maximum absorbance.

Text S2. Description of the ReaxFF force field

Figure S2. Time series of replicas at $300 \mathrm{~K}$ for REMD simulations of model $\# 1$ in water solvent at pH5

Figure S3. Probability distribution of potential energy at the first seven and the last five temperatures.

Figure S4. Charge transfer complex formed in model $3 \mathrm{pH} 5$ water solvent and calculated exited states characteristics

Table S2. Percentage of buried Carbon and Oxygen atoms of organic molecules in different solvents 


\section{Text S1. Chemical characteristics of SRFA used to build a model.}

In generating models, the molecular fragments that were detected by FTICR-MS in SRFA were used and were the most abundant components in the sample. This procedure allowed us to generate more realistic with greater flexibility than other tools (e.g., VSOMM). The molecular fragments designed by Leenheer at al., $1994^{1}$ were also used. In order to build the structures of SRFA, the following characteristics of SRFA were used:

1. Number-average molecular weight.

2. Elemental contents corrected for moisture and ash contents:

Carbon (C)

Hydrogen $(\mathrm{H})$

Oxygen (0)

Nitrogen $(\mathrm{N})$

Sulfur (S)

Phosphorus (P)

3. Average molecular formula.

4. Average moles of unsaturation $(\phi)$.

5. Carbon distribution by type of carbon:

Aliphatic

H-C-0 (alcohol, ether, ester, acetal, ketal)

0 -C-O (acetal, ketal) plus aromatic

Aromatic

Phenols, phenolic esters, aromatic ethers

Carboxyl plus ester

Ketone

6. Hydrogen distribution:

Exchangeable-hydrogen distribution by type of hydrogen:

Carboxyl

Phenol

Alcohol

Nonexchangeable hydrogen distribution by type of hydrogen:

Isolated aliphatic

$\mathrm{H}_{3} \mathrm{C}-\mathrm{C}=0, \mathrm{H}_{2} \mathrm{C}-\mathrm{C}=0$, H-C-C $=0, \mathrm{H}_{3}-\mathrm{C}-\phi, \mathrm{H}_{2}-\mathrm{C}-\phi, \mathrm{H}-\mathrm{C}-\phi, \mathrm{H}-\mathrm{C}-\mathrm{O}, \mathrm{H}-\phi$ 
7. Oxygen distribution by type of oxygen:

Carboxyl

Ester

Carboxyl+ester

Ketone

Phenol

Alcohol

Acetal and ketal

Ether

8. Amino acids

Metal-binding sites, nitrogencontaining functional groups

Metal-binding sites, sulfurcontaining functional groups

9. Organic free radicals

10. Metal-binding sites

Suwanee River fulvic acid was of particular interest due to the following factors: i) it been accepted as standard humic materials by the international scientific community, IHSS in particular, ii) there is a large body literature available, including extensive molecular level characterization, and iii) it is available to anyone in the international community at a very reasonable price 



Figure S1. Example of $\log D$ solubility calculation for a component, showing how this measure depends on the $\mathrm{pH}$ of the solution. $\log \mathrm{D}$ values were calculated using MarvinSketch ${ }^{2}$ 
Table S1. Molecular composition of the different models created and calculated values for $\operatorname{LogD}$ and maximum absorbance for individual molecules.

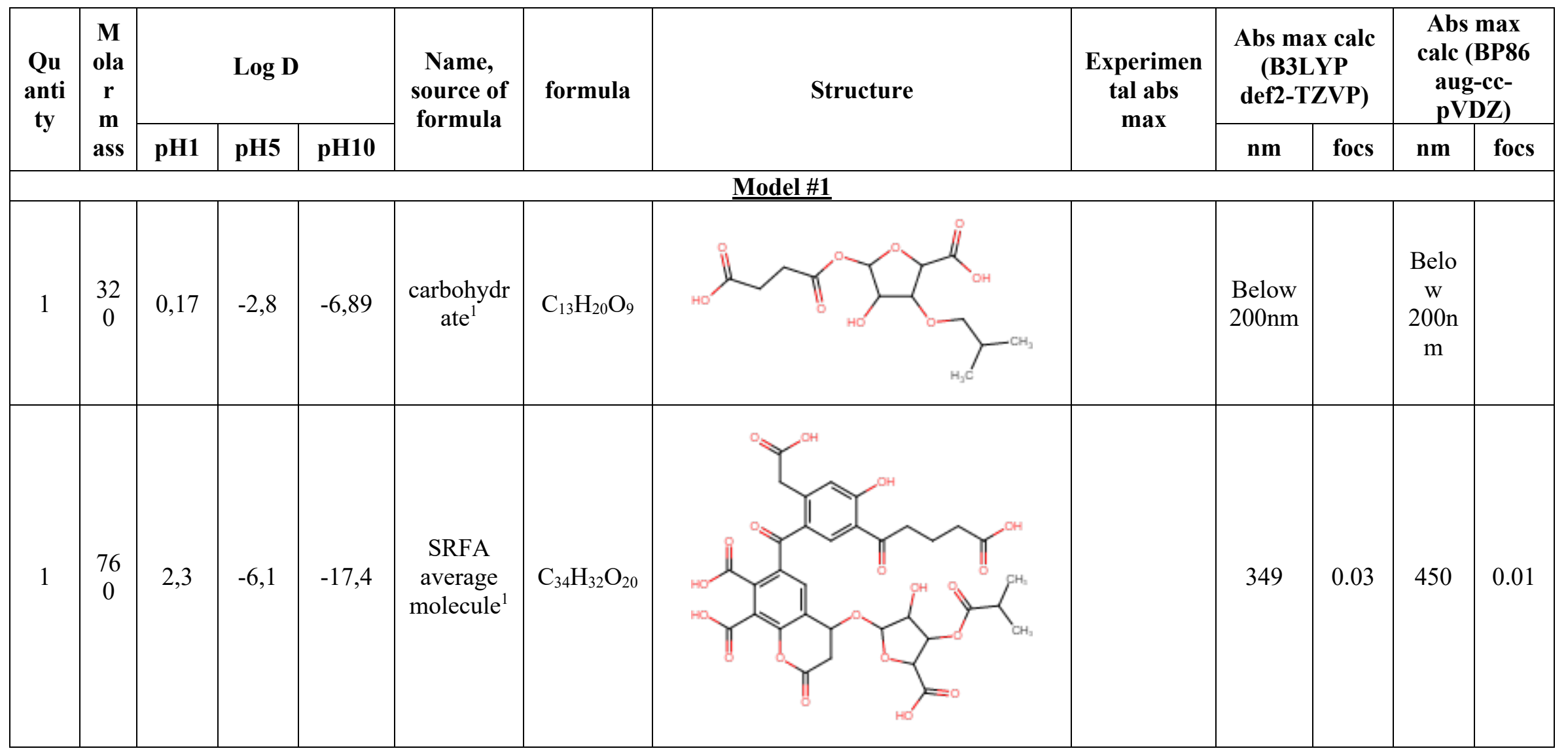




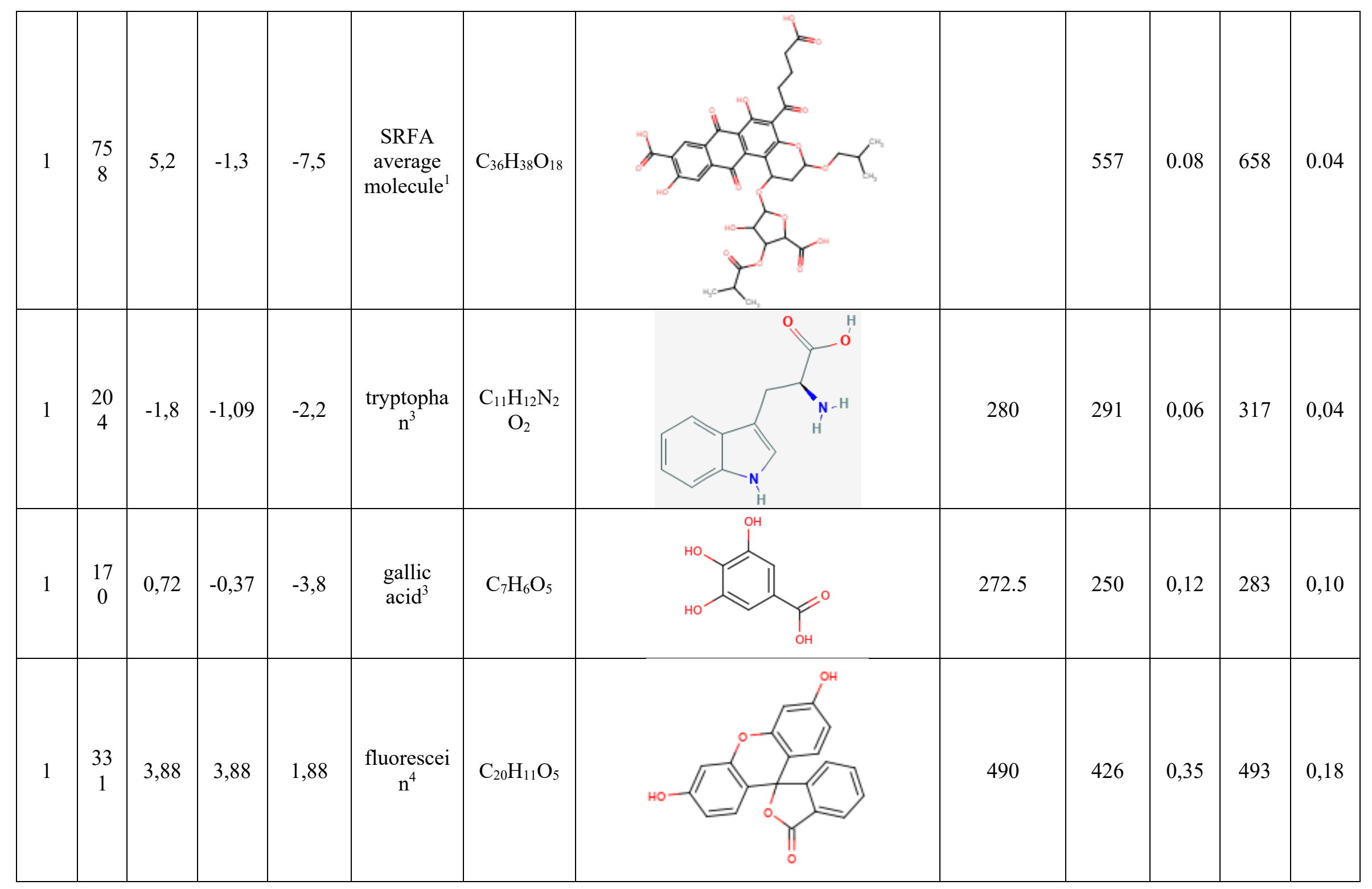




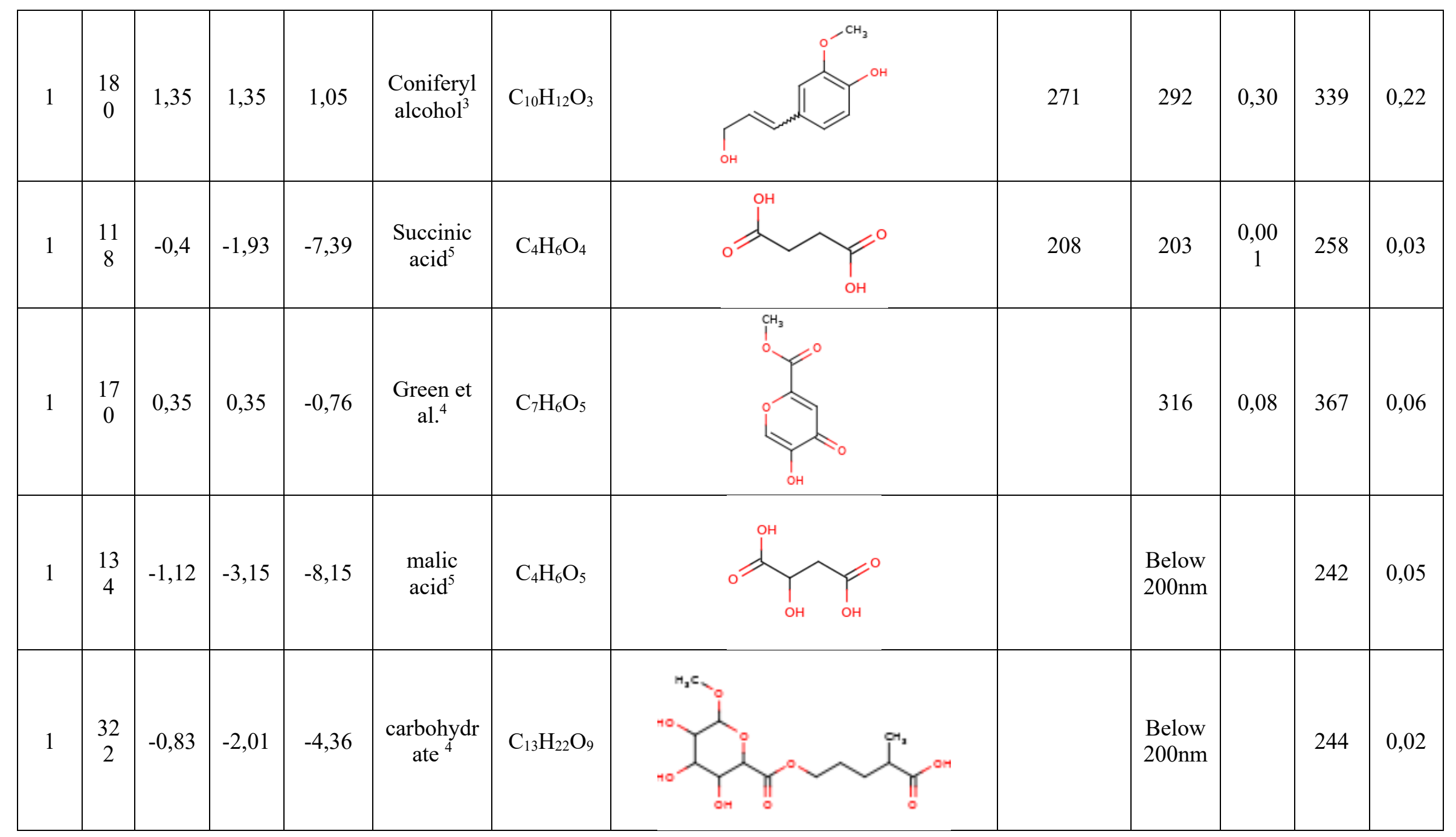




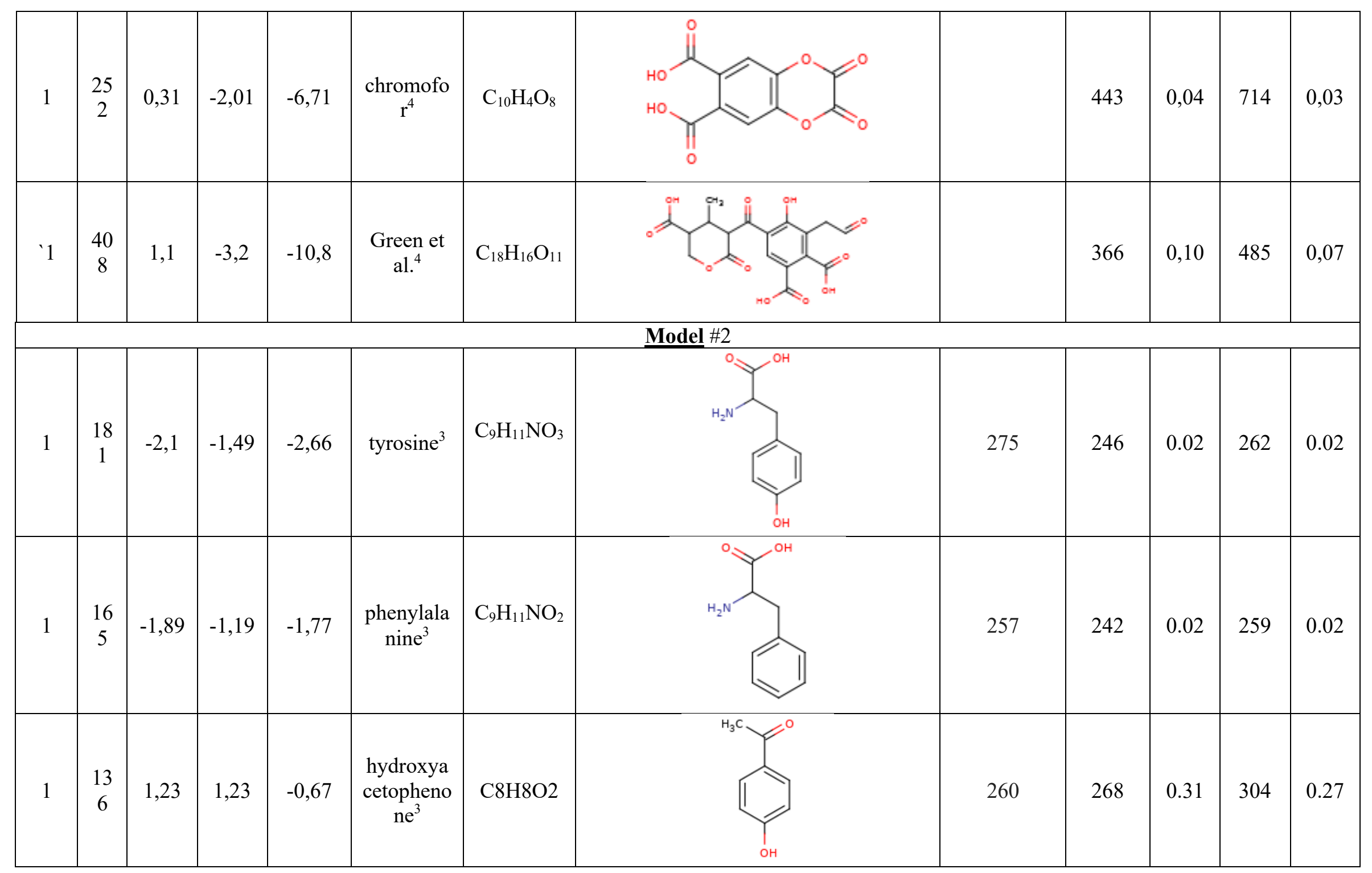




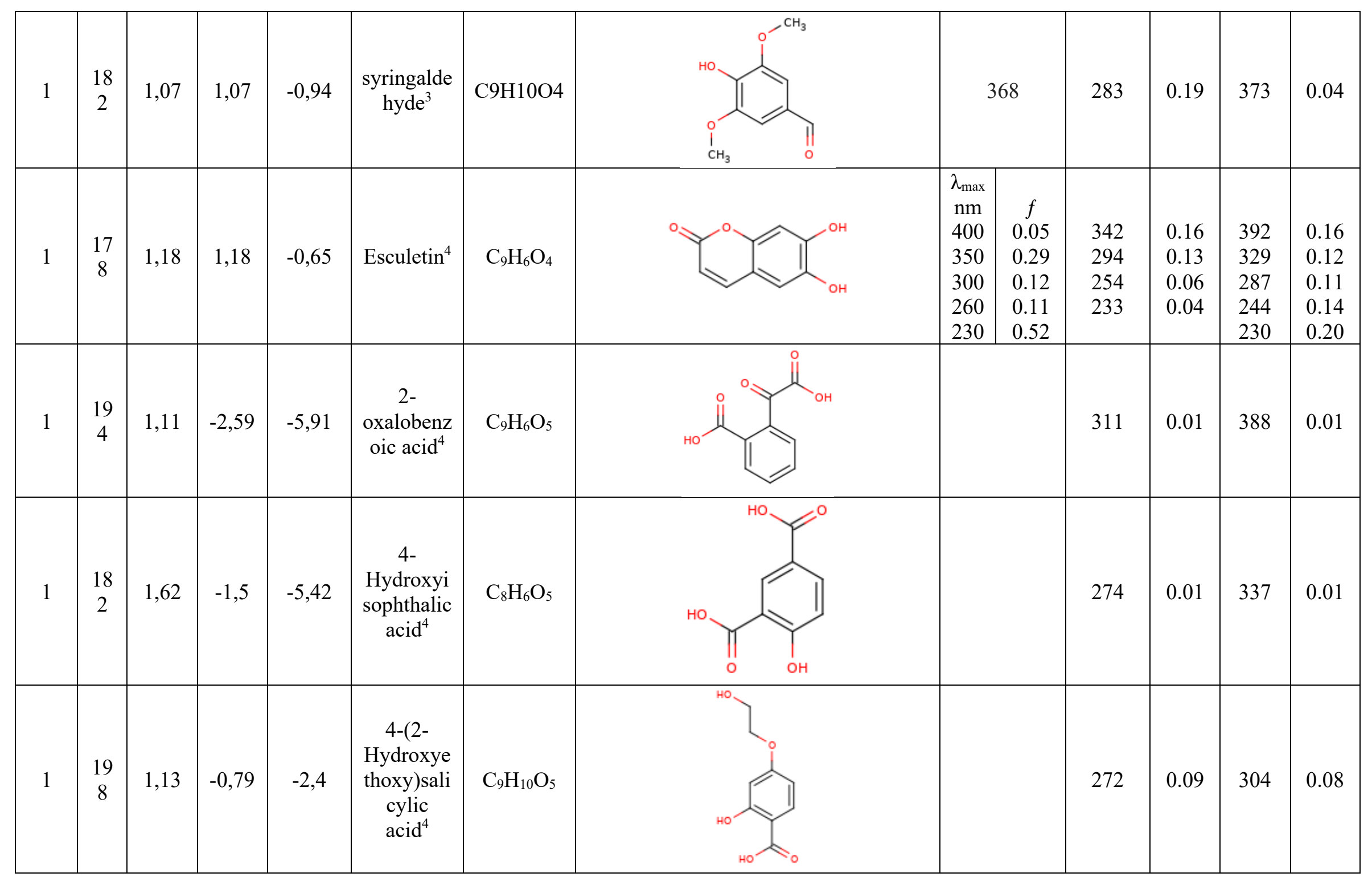




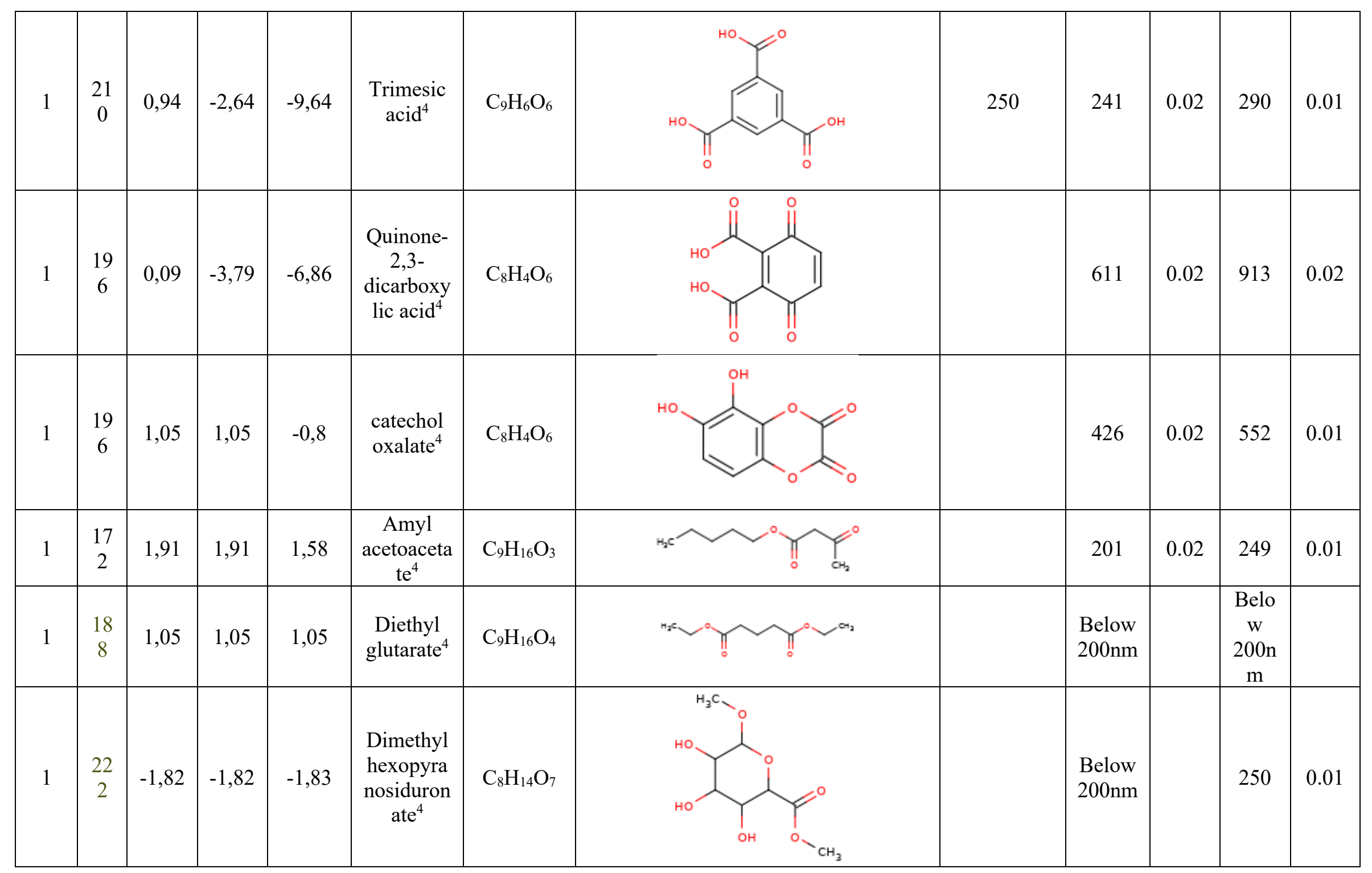




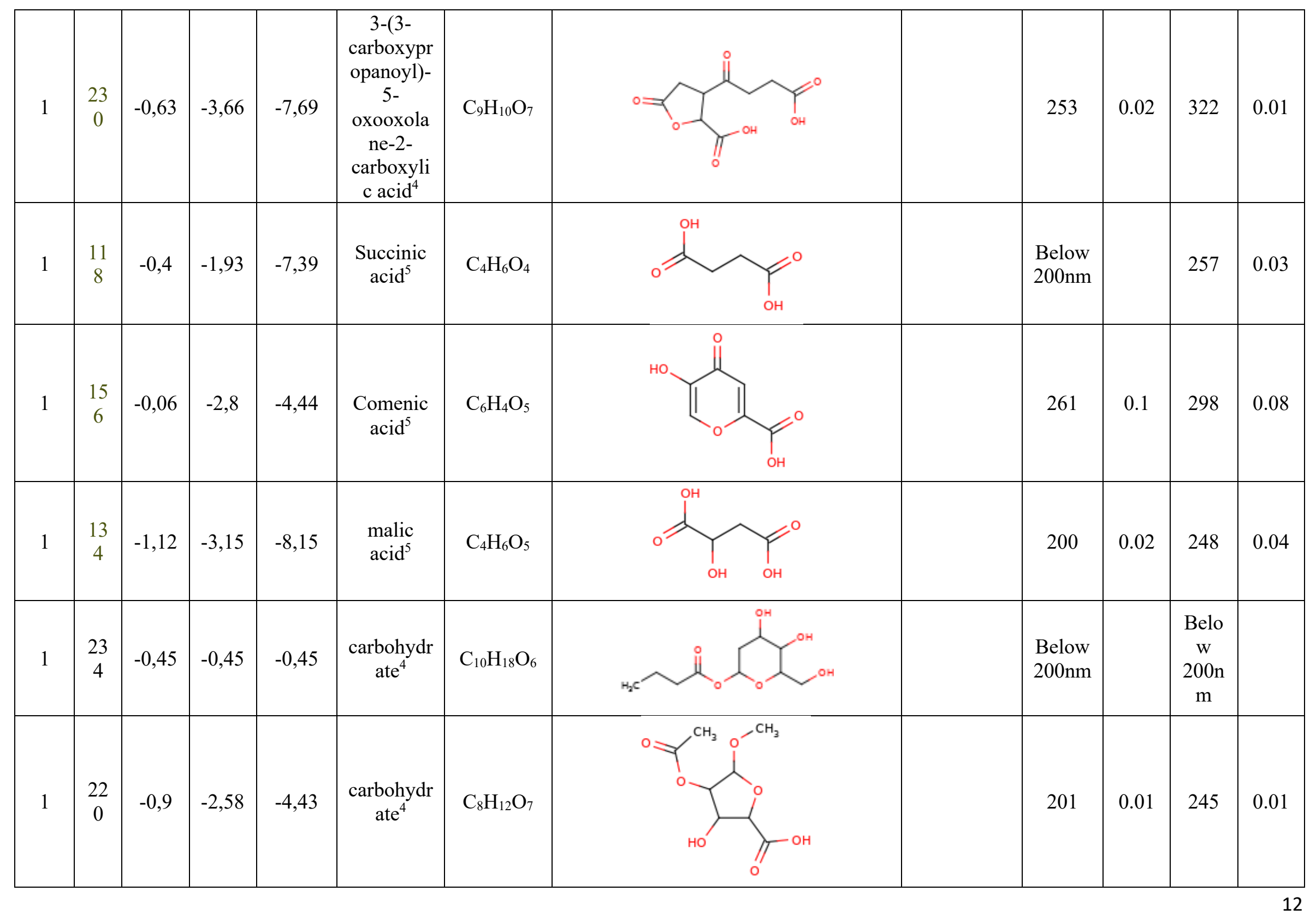




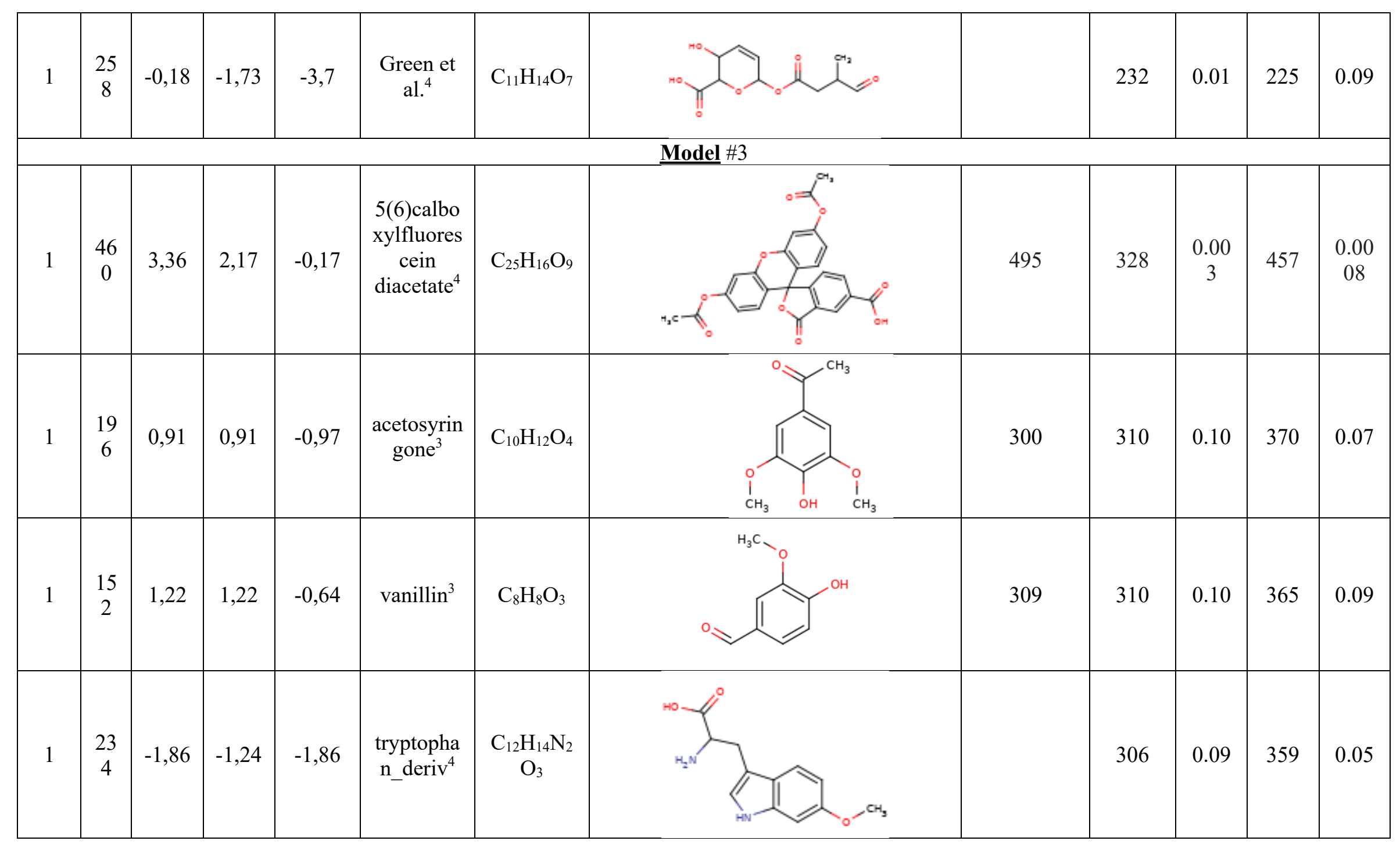









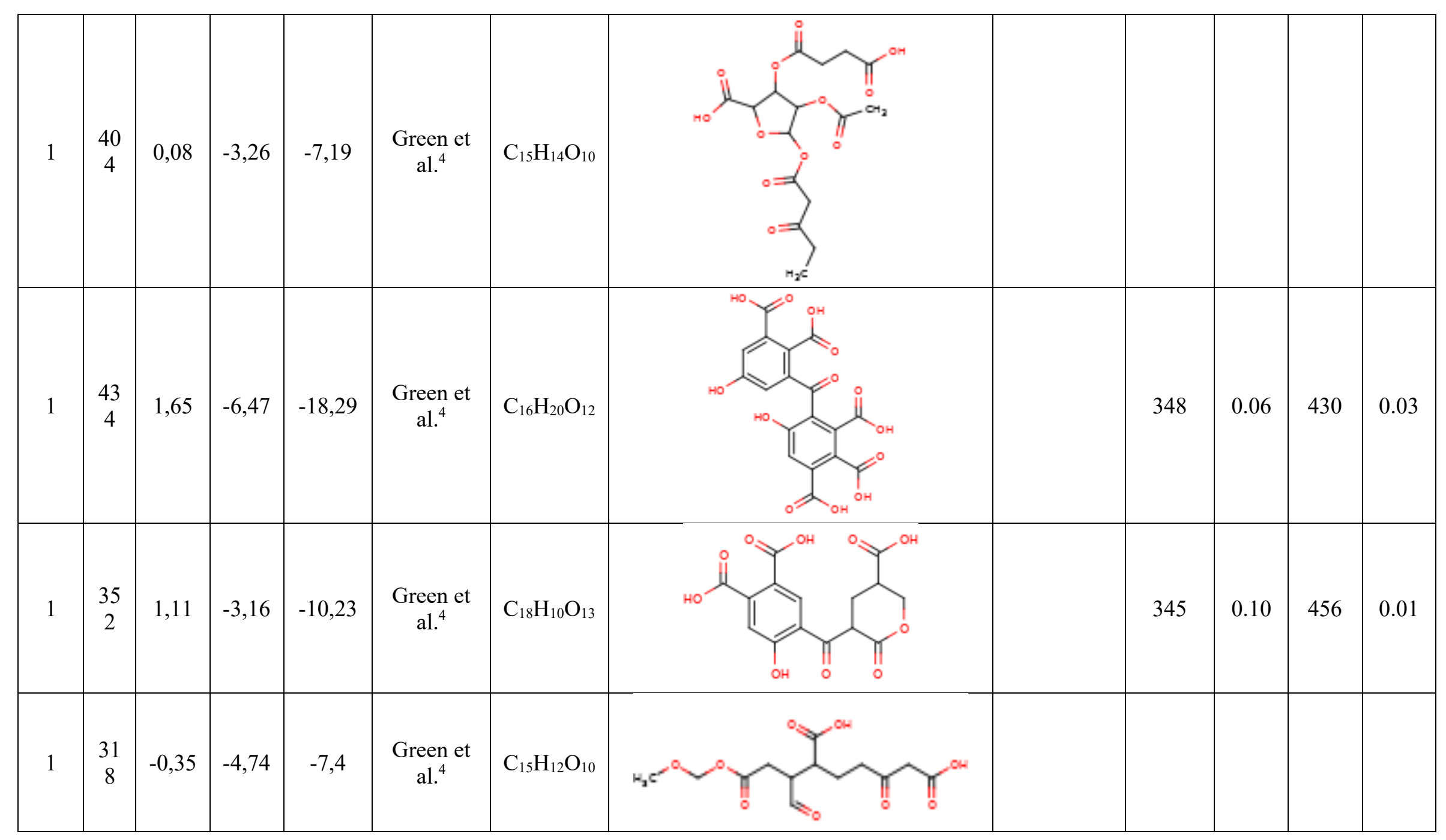




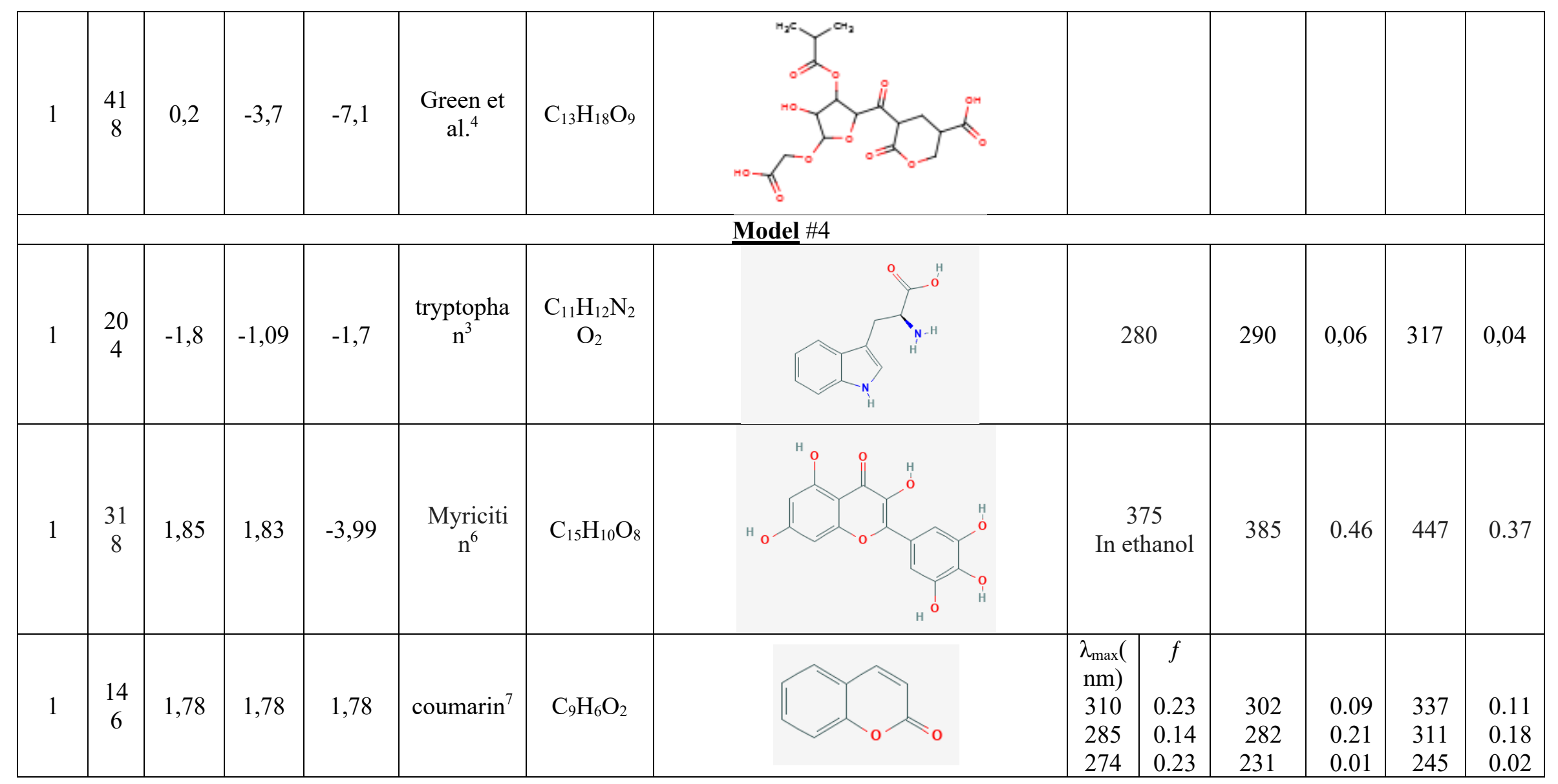









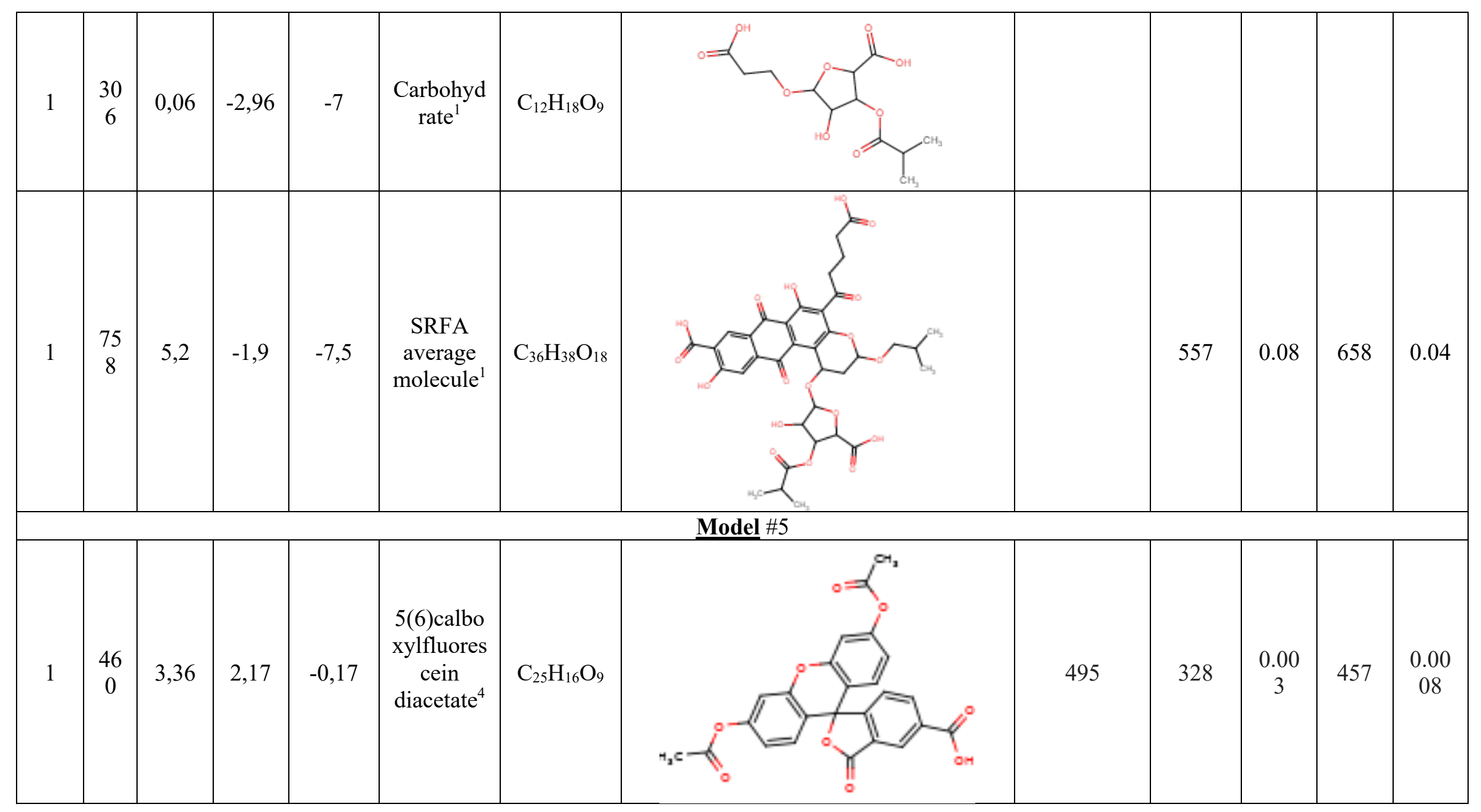




\begin{tabular}{|c|c|c|c|c|c|c|c|c|c|c|c|}
\hline 1 & $\begin{array}{c}23 \\
4\end{array}$ & $-1,86$ & $-1,24$ & $-1,86$ & $\begin{array}{c}\text { tryptopha } \\
\text { n_deriv }\end{array}$ & $\begin{array}{c}\mathrm{C}_{12} \mathrm{H}_{14} \mathrm{~N}_{2} \\
\mathrm{O}_{3}\end{array}$ & & 306 & 0.09 & 359 & 0.05 \\
\hline 1 & $\begin{array}{c}43 \\
4\end{array}$ & 0,78 & 0,76 & $-1,98$ & $\begin{array}{c}\text { Remucal } \\
\text { et al. }\end{array}$ & $\mathrm{C}_{19} \mathrm{H}_{14} \mathrm{O}_{12}$ & & 361 & 0.19 & 423 & 0.11 \\
\hline 1 & $\begin{array}{c}38 \\
4\end{array}$ & 1,37 & $-0,32$ & $-9,16$ & $\begin{array}{c}\text { Green et } \\
\text { al. }^{4}\end{array}$ & $\mathrm{C}_{18} \mathrm{H}_{24} \mathrm{O}_{9}$ & & & & & \\
\hline 1 & $\begin{array}{c}44 \\
2\end{array}$ & $-0,47$ & $-1,98$ & $-5,83$ & $\begin{array}{l}\text { Remucal } \\
\text { et al. }\end{array}$ & $\mathrm{C}_{18} \mathrm{H}_{18} \mathrm{O}_{13}$ & & 377 & 0.09 & 454 & 0.06 \\
\hline
\end{tabular}




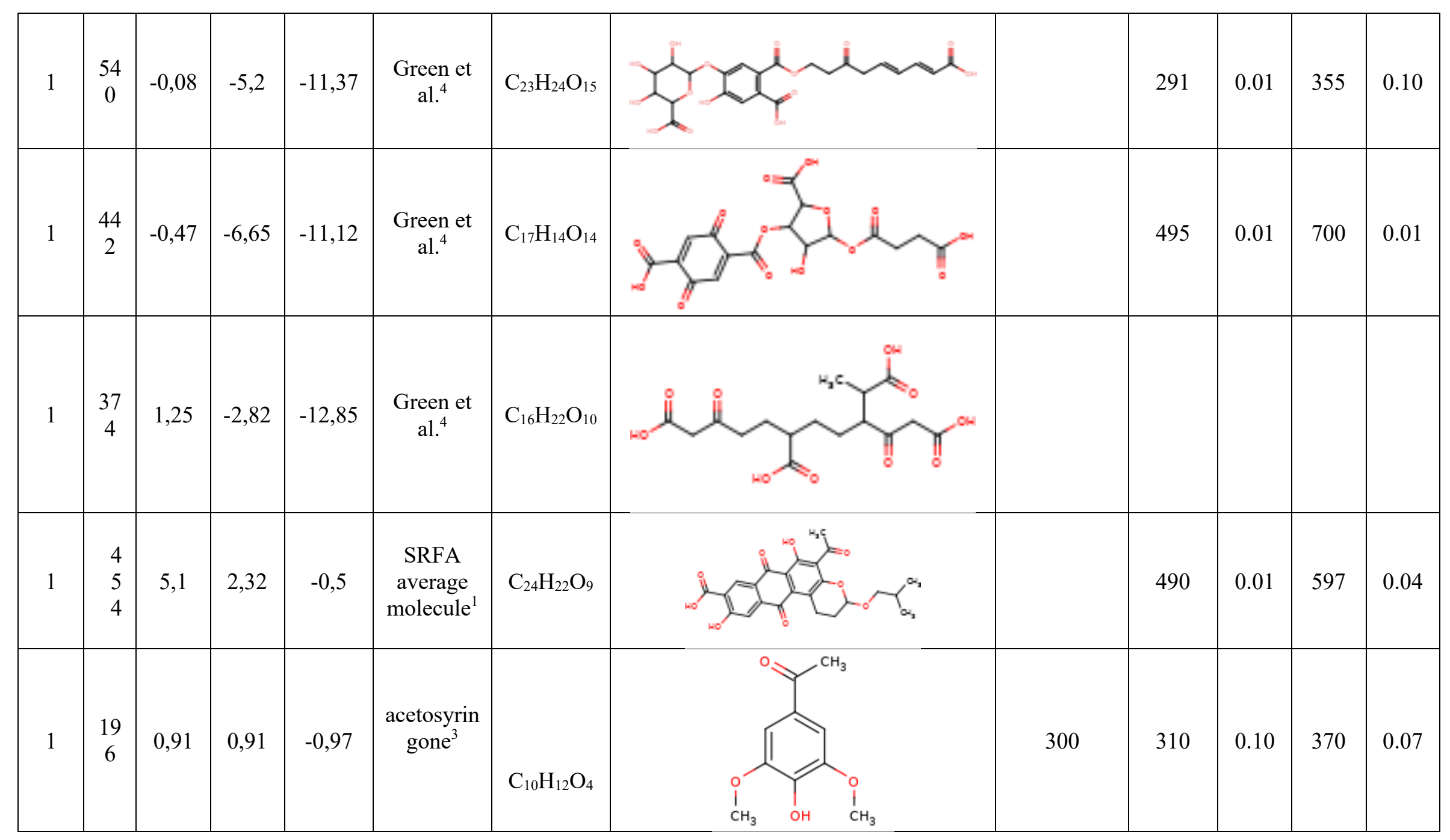




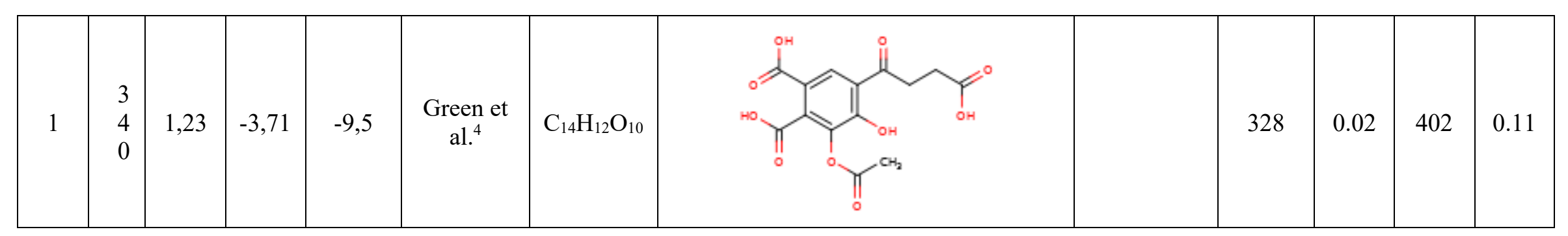




\section{Text S2. Description of the ReaxFF force field}

ReaxFF is a reactive force field that was used to simulate the various interactions, including 1) all connectivity-dependent interactions (i.e. valence and torsion angles) are made bond-order dependent, ensuring that their energy contributions disappear upon bond dissociation; 2) nonbonded interactions (van der Waals, Coulomb) are calculated between every atom pair, irrespective of connectivity. Excessive close-range nonbonded interactions are avoided by shielding; 3) ReaxFF uses a geometry-dependent charge calculation scheme that accounts for polarization effects. The force field is trained against a QM-derived set of energies for small molecules and clusters. For each element, several parameters to describe valence bond parameters, electronegativity, hardness, and other effects are optimized to reproduce the QM derived energies and charges. The electron equilibration method (EEM) is implemented to derive the changing atomic charges. This formulation enables polarization and charge transfer effects ${ }^{8}$.

The main advantage of ReaxFF is its ability to simulate covalent bond formation and breaking, i.e. primary chemical reactions of organic molecules. ReaxFF force field parameters are optimized to reproduce a suitable reference organic data set, derived mainly via quantum chemical calculations (QC) and compared to a number of QM/MM calculations (for more details please see ${ }^{9}$. Being empirical in nature, ReaxFF allows MD simulations of large reactive chemical systems (1000s of atoms) and yet retains an accuracy close to QC.

The ReaxFF water model is used to simulate aqueous solutions with explicit solvent. The parameters for the water model (molecule) are determined from quantum mechanics.

Since the HS of focus here consists of organic molecules in an aqueous environment, we used the ReaxFF potential developed to simulate biomolecules ${ }^{9}$.

In the word done by Vialykh et al. ${ }^{10}$, ReaxFF was tested for its ability to simulate molecular interactions that are important for complex organic mixtures and that occur in HS, including a) $\mathrm{H}$ bonding, (b) hydrophobic interactions, c) $\pi$-stacking, d) charge transfer complexation, e) labile metalion complexation, and $\mathrm{f}$ ) chemical reactions between molecular fragments. ReaxFF was used to study the functional behaviour of three different systems with organic contaminants, phenol, toluene and benzene, in the vacuum and aqueous phase, as well as complexation with $\mathrm{Cu}^{2+}$ in vacuum. A detailed description of the ReaxFF force field is presented in Vialykh et al. ${ }^{10}$ and includes the following:

In 2001 van Duin et al. ${ }^{8}$ developed a new force field (ReaxFF). The main advantage of ReaxFF is its ability to simulate covalent bond formation and breaking, i.e. primary chemical reactions of organic molecules. The general equation used in ReaxFF is: 


$$
E_{\text {system }}=E_{\text {bond }}+E_{\text {over }}+E_{\text {under }}+E_{l p}+E_{\text {val }}+E_{\text {tor }}+E_{\text {wdWaals }}+E_{\text {Coloumb }}
$$

It takes into account partial contributions to the total system potential energy $\left(E_{\text {system }}\right.$ related to the bond $\left(E_{\text {bond }}\right)$, over-coordination penalty $\left(E_{\text {over }}\right)$ and under-coordination stability $\left(E_{\text {under }}\right)$, lone pair $\left(E_{l p}\right)$, valence angle $\left(E_{v a l}\right)$ and torsion $\left(E_{\text {tor }}\right)$, and non-bonding Coulombic $\left(E_{\text {Coloumb }}\right)$ and van der Waals $\left(E_{\text {wdWaals }}\right)$ energies, respectively ${ }^{11}$.

The main assumption used in ReaxFF is that bond order $\left(B O_{\mathrm{ijj}}\right)$ can be derived directly from interatomic distance $\left(r_{i j}\right)$ according to equation (2):

$$
B O_{i l}^{\prime}=\exp \left[p_{b o, 1} *\left(\frac{r_{i j}}{r_{0}}\right)^{p_{b o, 2}}\right]+\exp \left[p_{b o, 3} *\left(\frac{r_{i j}{ }^{\pi}}{r_{0}}\right)^{p_{b o, 4}}\right]+\exp \left[p_{b o, 5} *\left(\frac{r_{i j}{ }^{\pi \pi}}{r_{0}}\right)^{p_{b o, 6}}\right]
$$

where $r_{o}$ is the bonding equilibrium distance. Three exponential terms used in equation (2) describe: 1) the sigma bond $\left(p_{b o, l}\right.$ and $\left.p_{b o, 2}\right)$ which is unity below $\sim 1.5 \AA$ but negligible above $\sim 2.5 \AA ; 2$ ) the first pi bond ( $p_{b o, 3}$ and $\left.p_{b o, 4}\right)$ which is unity below $\sim 1.2 \AA$ and negligible above $\sim 1.75 \AA$, and 3 ) the second pi bond ( $p_{b o .5}$ and $\left.p_{b o, 6}\right)$ which is unity below $\sim 1.0 \AA$ and negligible above $\sim 1.4 \AA$. As result a carbon-carbon bond has a maximum bond order of 3. For carbon-hydrogen and hydrogen-hydrogen bonds, only the sigma-bond contribution is considered, resulting in a maximum bond order of 1 . The use of two main relationships, bond distance/ bond order on the one hand and bond order/ bond energy on the other, allows modeling of bond dissociation and formation with ReaxFF.

The terms $E_{\text {over, }} E_{\text {under, }} E_{l p}$ are used to adjust bond order over-/under-coordination happening due to long-range interactions. When a carbon has a weak attraction/bond order with its second nearest neighbor, hydrogen atoms, this type of bonding will cause unrealistic behavior while modeling intact molecules, and must be corrected. In other words when a carbon atom has a bond order of 4 or more, these types of long-range interactions need to be negated and thus the small bond orders involving this carbon are significantly reduced to minimize their effects. Conversely, when a carbon atom has less than its optimal four bonds, these types of weak interactions should be allowed, so the weak bond orders are essentially unchanged. Consequently, these corrections allow accurate modeling of longrange radical attraction between atoms from different molecules as well as the realistic interactions between a radical site and its second nearest neighbors within the same molecule.

Since the bond orders are combined with functions of valence coordinates such as bond angles and torsion angles (equation 3) so that the energy contributions from bonding terms go to zero smoothly as bonds break. 


$$
E_{v a l}=\left[1-\exp \left(\lambda * B O_{1}^{3}\right)\right] *\left[1-\exp \left(\lambda * B O_{2}^{3}\right)\right] *\left\{k_{a}-k_{b} * \exp \left(-k_{b} *\left(\phi-\phi_{0}\right)^{2}\right)\right\}
$$

where $\mathrm{BO}_{1}$ and $\mathrm{BO}_{2}$ are the bond orders for each of the two bonds connecting the three atoms within an angle. $\lambda$ is an angular parameter set to obtain agreement with quantum calculated values, $k_{a}$ and $k_{b}$ are the harmonic force constants that determine the depth and width of the angular potential, respectively, $\phi$ is the angle, and $\phi_{0}$ is the equilibrium angle.

ReaxFF also allows the calculation of the polarization of charges within molecules (eq. 4):

$$
\frac{\partial E}{\partial q_{n}}=\chi_{n}+2 * q_{n} * \eta_{n}+C * \sum_{j=1} \frac{q_{j}}{\left\{r_{n j}^{3}+\left(1 / \gamma_{n j}\right)^{3}\right\}^{1 / 3}},
$$

In equation (4) $\chi_{n}$ is the electronegativity and $\eta_{n}$ is the hardness of element $n$ and $\gamma_{n j}$ is a shielding parameter between atoms $n$ and $j$. The charge values are dependent on the system geometry and determined for each time step of the simulation. ReaxFF uses a geometry-dependent charge calculation scheme that accounts for polarization effects.

The van der Waals and Coulomb forces are included from the beginning and calculated (eq. 5) between every atom pair which allows the description of non-bonded interactions between all atoms.

$$
E_{\text {Coulomb }}=C *\left[\frac{q_{i} * q_{j}}{\left\{r_{i j}^{3}+\left(1 / \gamma_{i j}\right)^{3}\right\}^{1 / 3}}\right]
$$

where $q_{i}$ and $q_{j}$ are the charges of the two atoms, $r_{i j}$ is the interatomic distance and $C$ is the electric constant, and $\gamma_{i j}$ is the shielding parameter between atoms $i$ and $j$. Atomic charges are calculated using the Electron Equilibration Method approach.

Parameters for the dissociation and reaction curves are derived from quantum chemical calculations, thus ReaxFF allows molecular dynamics simulations of large-scale reactive chemical systems (1000s of atoms) with resulting accuracy similar to quantum mechanically based methods. Each element is represented by only 1 atom type in the force field; the force field should be able to determine equilibrium bond lengths, valence angles etc. from the chemical environment. 


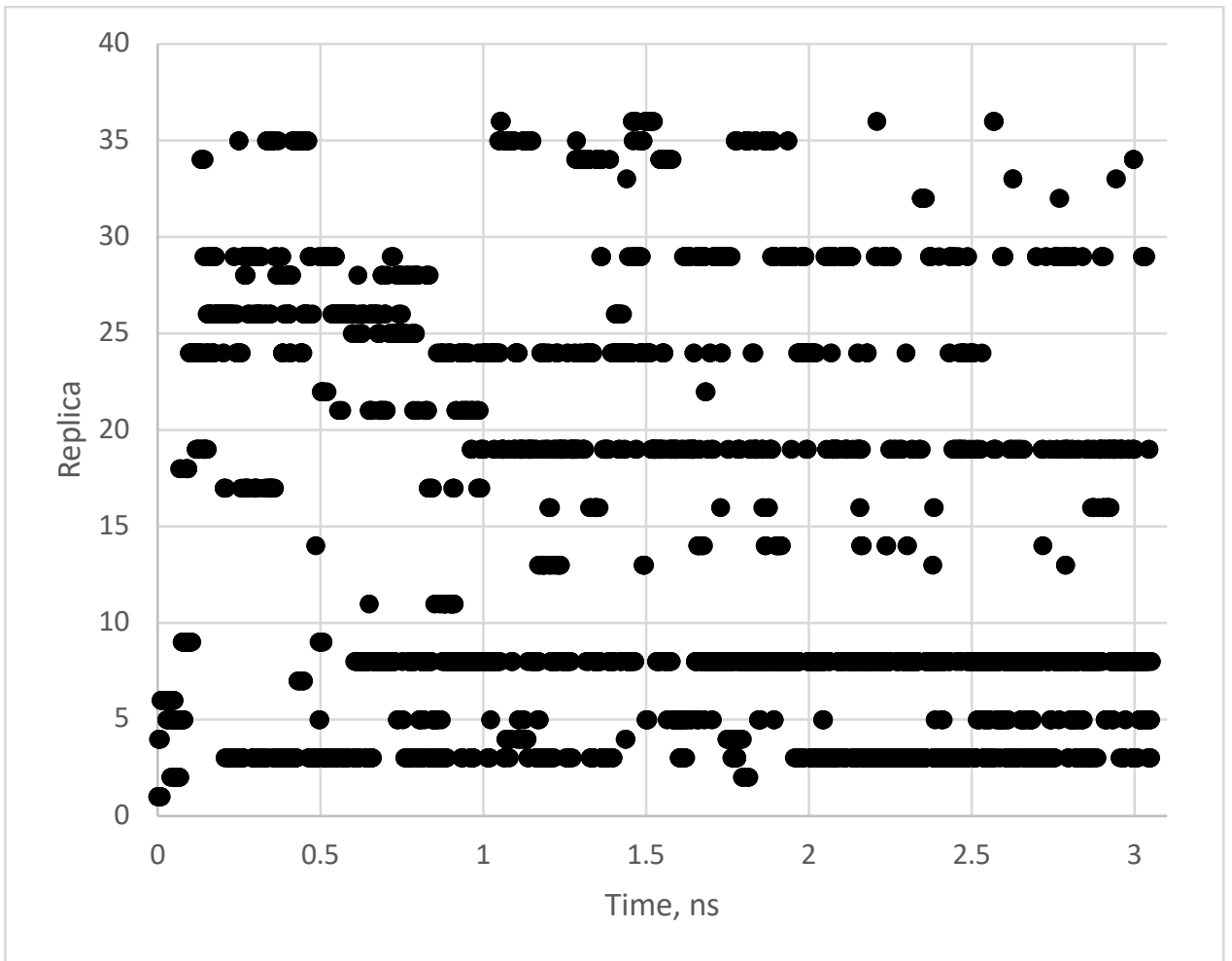

Figure S2. Time series of replicas at $300 \mathrm{~K}$ for REMD simulations of model \#1 in water solvent at pH5 




Figure S3. Probability distribution of potential energy at the first seven and the last five temperatures. Overlap between the distributions facilitates efficient exchange.

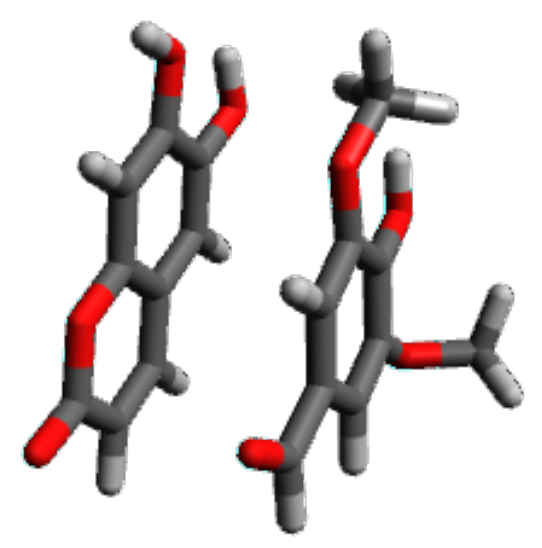

\begin{tabular}{|l|l|l|l|}
\hline state & $\Delta \mathrm{E}, \mathrm{eV}$ & $q(\mathrm{CT})$ & $f$ \\
\hline $\mathrm{S}_{1}$ & 2.059 & 0.96 & 0.008 \\
\hline $\mathrm{S}_{2}$ & 2.287 & 0.96 & 0.003 \\
\hline
\end{tabular}

Figure S4. Charge transfer complex formed in model $3 \mathrm{pH} 5$ water solvent and calculated exited states characteristics: vertical excitation energy $(\Delta \mathrm{E})$, charge transfer value $q(\mathrm{CT})$, and oscillator strengths $(f)$. The charge transfer character (CT) is defined as: $C T=\frac{1}{\Omega} \sum_{B \neq A} \Omega_{A B}$. CT assumes the value 1 for all completely charge separated states, whereas it is 0 for locally excited or Frenkel excitonic states ${ }^{12}$. 
Table S2. Percentage of buried Carbon and Oxygen atoms (atoms that inaccessible to the $1.4 \AA$ water probe) of organic molecules in different solvents.

\begin{tabular}{|c|c|c|c|}
\hline $\begin{array}{c}\text { Model } \\
\#\end{array}$ & solvent & $\%$ of buried $\mathrm{C}$ atoms & $\%$ f buried $\mathrm{O}$ atoms \\
\hline \multirow{5}{*}{1} & Water pH5 & 17.1 & 0.04 \\
\hline & Water pH10 & 6.4 & 0 \\
\hline & MTBE & 2.1 & 0 \\
\hline & THF & 4.3 & 0.04 \\
\hline & $3000 \mathrm{WM}$ pH5 & 7.5 & 0.01 \\
\hline \multirow{4}{*}{2} & Water $\mathrm{pH} 5$ & 5.2 & 0.03 \\
\hline & Water pH10 & 3.5 & 0.01 \\
\hline & MTBE & 0 & 0 \\
\hline & THF & 0 & 0 \\
\hline \multirow{4}{*}{3} & Water pH5 & 10.0 & 0.02 \\
\hline & Water pH10 & 5.8 & 0.02 \\
\hline & MTBE & 3.2 & 0 \\
\hline & THF & 3.2 & 0 \\
\hline \multirow{4}{*}{4} & Water $\mathrm{pH} 5$ & 13.7 & 0.03 \\
\hline & Water pH10 & 8.7 & 0.01 \\
\hline & MTBE & 1.6 & 0 \\
\hline & THF & 1.1 & 0.01 \\
\hline \multirow{4}{*}{5} & Water $\mathrm{pH} 5$ & 19.4 & 0.05 \\
\hline & Water $\mathrm{pH} 10$ & 8.2 & 0.04 \\
\hline & MTBE & 1.0 & 0 \\
\hline & THF & 1.0 & 0 \\
\hline
\end{tabular}




\section{References}

(1) Leenheer, J. A.; McKnight, D. M.; Thurman, E. M.; MacCarthy, P. Structural Components and Proposed Structural Models of Fulvic Acid from the Suwannee River. In Humic Substances in the Suwannee River, Georgia: Interactions, Properties, and Proposed Structures; Averett, R. C., Leenheer, J. A., McKnight, D. M., Thorn, K. A., Eds.; US Geological Servey, No 87-557; U.S. G.P.O. ; U.S. Geological Survey, Map Distribution, 1994; pp 195-212.

(2) ChemAxon - Software Solutions and Services for Chemistry \& Biology https://chemaxon.com/ (accessed Jul 27, 2020).

(3) Wünsch, U. J.; Murphy, K. R.; Stedmon, C. A. Fluorescence Quantum Yields of Natural Organic Matter and Organic Compounds: Implications for the Fluorescence-Based Interpretation of Organic Matter Composition. Front. Mar. Sci. 2015, 2. https://doi.org/10.3389/fmars.2015.00098.

(4) Green, N. W.; Perdue, E. M. Fast Graphically Inspired Algorithm for Assignment of Molecular Formulae in Ultrahigh Resolution Mass Spectrometry. Anal. Chem. 2015, 87 (10), 5086-5094. https://doi.org/10.1021/ac504166t.

(5) Remucal, C. K.; Cory, R. M.; Sander, M.; McNeill, K. Low Molecular Weight Components in an Aquatic Humic Substance As Characterized by Membrane Dialysis and Orbitrap Mass Spectrometry. Environ. Sci. Technol. 2012, 46 (17), 9350-9359.

https://doi.org/10.1021/es302468q.

(6) Qin, C.; Xie, M.-X.; Liu, Y. Characterization of the Myricetin-Human Serum Albumin Complex by Spectroscopic and Molecular Modeling Approaches. Biomacromolecules 2007, 8 (7), 2182-2189. https://doi.org/10.1021/bm070319c.

(7) Grimm, J. B.; Heckman, L. M.; Lavis, L. D. The Chemistry of Small-Molecule Fluorogenic Probes. In Progress in Molecular Biology and Translational Science; Elsevier, 2013; Vol. 113, pp 1-34. https://doi.org/10.1016/B978-0-12-386932-6.00001-6.

(8) van Duin, A. C. T.; Dasgupta, S.; Lorant, F.; Goddard, W. A. ReaxFF: A Reactive Force Field for Hydrocarbons. J. Phys. Chem. A 2001, 105 (41), 9396-9409. https://doi.org/10.1021/jp004368u.

(9) Monti, S.; Corozzi, A.; Fristrup, P.; Joshi, K. L.; Shin, Y. K.; Oelschlaeger, P.; van Duin, A. C. T.; Barone, V. Exploring the Conformational and Reactive Dynamics of Biomolecules in 
Solution Using an Extended Version of the Glycine Reactive Force Field. Phys. Chem. Chem. Phys. 2013, 15 (36), 15062-15077. https://doi.org/10.1039/c3cp51931g.

(10) Vialykh, E. A.; Salahub, D. R.; Achari, G.; Cook, R. L.; Langford, C. H. Emergent Functional Behaviour of Humic Substances Perceived as Complex Labile Aggregates of Small Organic Molecules and Oligomers. Environ. Chem. 2019, 16 (7), 505.

https://doi.org/10.1071/EN19095.

(11) Russo, M. F.; van Duin, A. C. T. Atomistic-Scale Simulations of Chemical Reactions: Bridging from Quantum Chemistry to Engineering. Nucl. Instrum. Methods Phys. Res. Sect. B Beam Interact. Mater. At. 2011, 269 (14), 1549-1554.

https://doi.org/10.1016/j.nimb.2010.12.053.

(12) Plasser, F.; Lischka, H. Analysis of Excitonic and Charge Transfer Interactions from Quantum Chemical Calculations. J. Chem. Theory Comput. 2012, 8 (8), 2777-2789. https://doi.org/10.1021/ct300307c. 